\title{
Control of Cable Actuated Devices using Smooth Backlash Inverse
}

\author{
Varun Agrawal, William J. Peine, Bin Yao and SeungWook Choi
}

\begin{abstract}
Cable conduit actuation provides a simple yet dexterous mode of power transmission for remote actuation. However, they are not preferred because of the nonlinearities arising from friction and cable compliance which lead to backlash type of behavior. Unlike most of the current research in backlash control which generally assumes no knowledge of one of the intermediate states, the controller design in this case can be significantly simplified if output feedback of the system is available. This paper uses a simple feedforward control law for backlash compensation. A novel smooth backlash inverse is proposed, which takes the physical limitations of the actuator in consideration, unlike other designs, and thus makes it more intuitive to use. Implementation of this inverse on physical systems can also improve the system performance over the theoretical exact inverse, as well as other existing smooth inverse designs. Improvement in the performance is shown through experiments on a robot arm of Laprotek surgical system as well as on an experimental setup using polymeric cables for actuation.
\end{abstract}

\section{INTRODUCTION}

$\mathrm{N}$ ONLINEARITIES due to cable compliance and friction are one of the biggest deterrents in using cable conduit system for actuation in applications where remote power transmission is required. As a result, rigid cable pulley systems are often preferred over flexible cable conduits to actuate the tools despite their numerous advantages [1], [2]. Cable conduits can pass through long narrow pathways and can easily endure the tortuous paths in applications like catheters. Furthermore, it can be implemented in very small working envelope and thus help in reducing the size of the overall system considerably. It drastically simplifies the transmission and also has the added advantage of being cost effective. Due to its simplicity of design, it is used for high manipulability and power dense systems, like colonoscopy [3], laparoscopic surgical robots, [4], [5], exoskeletons [6], [7], and some robotic hands [8] - [10].

However, all these advantages are overshadowed by the nonlinear behavior caused by the interaction between cable compliance and friction within the conduit. This problem aggravates significantly in the surgical robots where long cables are used, passing through sharp curves and multiple loops; especially in applications like catheters and colonoscopes. Friction and cable compliance levels become

Manuscript received September 15, 2009. This work was supported by Meere Company, Republic of Korea.

V. Agrawal, W. J. Peine, B.Yao are with School of Mechanical Engineering, Purdue University, West Lafayette, IN 47906 USA (e-mail: vagrawal, peine, byao @ purdue.edu, phone: 765-494-7746).

S. Choi is with Rebo Co. Ltd., Gyeonggi, Republic of Korea (e-mail: samuelchoi@surgicalrobot.biz) significantly higher and lead to nonlinearities in the system, like backlash, cable slacking and input dependent stability of the system [11], [12], [13]. These nonlinearities make it unfavorable for a surgeon to operate a surgical system without any closed loop control. Various techniques are adopted in practice to keep the friction effects at low levels, e.g., using PTFE-coated steel cables sliding in slightlypreloaded Kevlar-reinforced housings, keeping the cablewrapping angles to low levels etc. [14]. These hardwarebased measures can reduce the friction levels only to a certain degree. Beyond that, one has to rely on effective control algorithms to remove the need for surgeon adaptation, which the state-of-the-art and current research lacks. Successful implementation of effective controllers offers a path for tremendous advancement in the development of cheaper and better surgical and other robotic devices. This paper presents a feedforward controller using smooth backlash inverse for such systems.

Various research works have been carried out to quantify the effect of these nonlinearities in cable conduit systems. Kaneko et al. [15], [16], and Palli and Melchiorri [17], [18] modeled these nonlinearities for a single cable actuation, typically used in robotic hand. Although, corresponding control law were proposed by Palli and Melchiorri it assumed knowledge of physical parameters of the system which is not possible in most practical scenarios. The transmission characteristics for cable-conduits in pull-pull configuration were experimentally studied by Kaneko et al [11]. In earlier work by the authors, an analytical model for transmission using pull-pull configuration [13], [19] was developed. It was shown that the effects due to friction eventually lead to a backlash type of behavior in the system. Thus, as a first cut for controller design, it is reasonable to model the load transmission in the system as an actuator in series with a backlash at the output.

Backlash is a common nonlinearity present in a wide range of mechanical systems including actuators and sensors, hydraulic servo valves, piezoelectric translators, electronic relay circuits and electric servomotors, and thus, has been a focus of significant amount of research [20] - [22]. Some of the recent important developments in control of systems with backlash have been basically based on perfect direct inversion of piecewise linearly parametrized deadzone and backlash nonlinearities, pioneered by Tao and Kokotovic in a series of papers and books [20], [23], [24]. Through the use of exact inverse, various sophisticated adaptive control algorithms have been developed which solve the problem theoretically, guaranteeing global stability and certain 
transient performances [20], [21]. However, very little experimental work has been reported. The use of inverse mappings involves discontinuous switching of control inputs, and thus is significantly restricted by actuator bandwidth limitations. To overcome this problem, although smooth inverse has been proposed by Zhou et al [21], it lacks the effective use of system information, and thus appears another step in solving a problem theoretically.

In most cable conduit actuated systems, together with the availability of the motion measurement of the actuating joint, the motion of the output joint can also be measured using sensors or other motion estimation processes. This leads to a simplified backlash control problem since both input and output to the backlash are known, as opposed to the cases when one of the states are unknown [20], [21]. This paper presents a smooth backlash inverse to compensate for the nonlinearity. The smoothing has been designed, such that it can easily be adapted to the actuator bandwidth limitations without affecting the system stability. This smooth inverse has been implemented on two different systems to show its usability, a surgical robot arm designed for a laparoscopic robot, and an experimental setup using polymeric cables. For the backlash inverse, online least squares approach has been used for parameter adaptation to account for slow changes in backlash parameters, as observed in the case of viscoelastic polymer cables.

\section{BACKLASH INVERSE}

\section{A. Backlash Characteristics}

Backlash nonlinearity from input $v(t)$ to output $u(t)$, as shown in Fig. 1, can be mathematically represented as

$$
\begin{aligned}
& u(t)=\mathcal{B}(v(t))=\mathcal{B}\left(m, c_{r}, c_{l} ; v(t)\right) \\
& = \begin{cases}m\left(v(t)-c_{r}\right) & \text { if } \dot{v}(t)>0 \text { and } u\left(t^{-}\right)=m\left(v\left(t^{-}\right)-c_{r}\right) \\
m\left(v(t)-c_{l}\right) & \text { if } \dot{v}(t)<0 \text { and } u\left(t^{-}\right)=m\left(v\left(t^{-}\right)-c_{l}\right) \\
u\left(t^{-}\right) & \text {otherwise. }\end{cases}
\end{aligned}
$$

where, $m$ denotes the slope of the backlash, and $c_{r}$ and $c_{l}$ denote the offsets on the right and left side respectively. The region when the output does not change with the change in input, i.e. $u(t)=u\left(t^{-}\right)$, can be interpreted as temporary loss of controllability of the system.

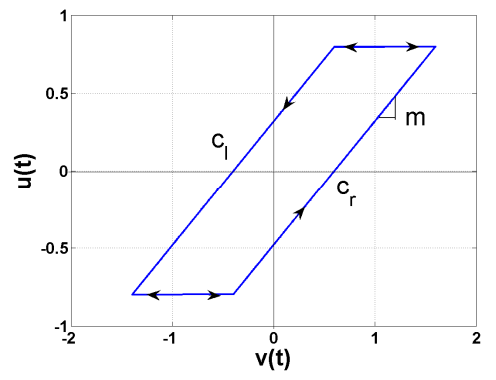

Fig. 1: Backlash model

\section{B. Backlash Inverse}

Tao and Kokotovic [20] first defined the concept of backlash inverse as an inversion of the backlash nonlinearity. When preceding the backlash, it can completely cancel the nonlinearity if the backlash parameters are precisely known, shown by the solid blue line in Fig. 2 and defined as:

$$
\begin{aligned}
v(t) & =\mathcal{B I}\left(u_{d}(t)\right)=\mathcal{B I}\left(\hat{m}, \hat{c}_{r}, \hat{c}_{l} ; u_{d}(t)\right) \\
& = \begin{cases}\frac{u_{d}(t)}{\hat{m}}+\hat{c}_{l} & \dot{u}_{d}(t)<0 \\
\frac{u_{d}(t)}{\hat{m}}+\hat{c}_{r} & \dot{u}_{d}(t)>0\end{cases}
\end{aligned}
$$

where, $\hat{m}, \hat{c}_{r}, \hat{c}_{l}$ denote the parameter estimates of $m, c_{r}, c_{l}$. Since the nonlinearity is inherently non-smooth, the inverse also is discontinuous, and cannot be physically realized. To resolve this issue, Tao and Kokotovic suggested using a smoothing function, but no specific form was reported. Later on, Zhou et al [21] proposed a smooth inverse defined as:

$$
\begin{aligned}
& v(t)=\frac{1}{\hat{m}} u_{d}(t)+\chi_{r}\left(\dot{u}_{d}\right) \hat{c}_{r}(t)+\chi_{l}\left(\dot{u}_{d}\right) \hat{c}_{l}(t) \\
& \chi_{r}=\frac{e^{k \dot{u}_{d}}}{e^{k \dot{u}_{d}}+e^{-k \dot{u}_{d}}} ; \quad \chi_{l}=\frac{e^{-k \dot{u}_{d}}}{e^{k \dot{u}_{d}}+e^{-k \dot{u}_{d}}}=1-\chi_{r}
\end{aligned}
$$

such that, $\chi_{r}\left(\dot{u}_{d}\right) \rightarrow 1$ as $\dot{u}_{d} \rightarrow \infty$, and so on. However, the logic behind the design of this function was not explained. This smoothing function does not take the actuator dynamics into account, and the choice of the gain $k$ appears to be arbitrary or at best determined by tuning. Tuning of gain $k$ will be governed by two issues, none of which can be easily addressed. First, the tuning will be trajectory specific, and a system tuned to certain peak velocity levels will not perform well at lower or higher operating velocities. Second, since $\dot{u}_{d}$ is generated by the control law, it is not available apriori. Therefore, tuning of $k$ is not possible before controller design. Moreover, the smoothing function is dependent on velocity and thus is highly prone to measurement noise, leading to chattering at low velocities.

For all practical applications, since $\dot{u}_{d}$ is finite, the indicator functions $\chi_{r}, \chi_{l}$ never approach 0 or 1 and at any time instant both $\chi_{r}, \chi_{l}$ have finite values. Therefore, although this smooth inverse might seem to work theoretically, in practice, it can lead to a trajectory far from the exact inverse, as shown by the dashed red line in Fig. 2. Furthermore, this function smoothes out all the four corners of the inverse, as if smoothing in anticipation, which is not necessary for practical implementation as discussed later, and introduces unnecessary errors due to increased digression from the exact inverse. 


\section{Proposed smooth inverse}

The inverse in (2) and (3) is only defined for $\dot{u}_{d}>0$ and $\dot{u}_{d}<0$, and no consideration has been given for the case of $\dot{u}_{d}=0$. Small variations in $\dot{u}_{d}$ close to zero can lead to continuous switching between the two states of the inverse. To avoid that chattering, the inverse should be modified to:

$$
\begin{aligned}
v(t) & = \begin{cases}\frac{u_{d}(t)}{\hat{m}}+\hat{c}_{l} & \dot{u}_{d}(t)<0 \\
\frac{u_{d}(t)}{\hat{m}}+\hat{c}_{r} & \dot{u}_{d}(t)>0 \\
v\left(t^{-}\right) & \dot{u}_{d}(t)=0\end{cases} \\
& =\frac{u_{d}(t)}{\hat{m}}+u_{s}(t)
\end{aligned}
$$

where, $u_{d}(t) / \hat{m}$ denotes the direct transmission, and $u_{s}(t)$ represents the discontinuous jumps in the trajectory defined as:

$$
u_{s}(t)= \begin{cases}\hat{c}_{l} & \dot{u}_{d}(t)<0 \\ \hat{c}_{r} & \dot{u}_{d}(t)>0 \\ u_{s}\left(t^{-}\right) & \dot{u}_{d}(t)=0\end{cases}
$$

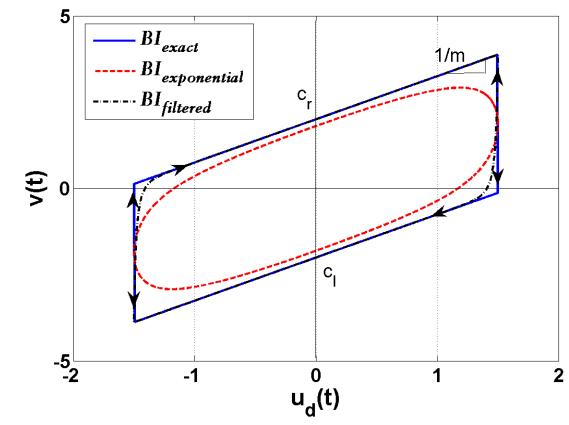

Fig. 2: Backlash inverse model for: (i) Exact Inverse, (ii) Smooth inverse designed by Zhou et al., and (iii) Proposed smooth inverse

Since, the motivation behind designing a smooth inverse is to address the physical limitations of the actuator, instead of designing any smoothing function; the most intuitive approach will be to limit the required bandwidth to follow the inverse. Therefore, we propose to use a second order filter for smoothing, as shown in Fig. 3. To minimize the lag introduced due to filter, only the $u_{s}(t)$ component of the inverse is filtered. The parameters of the filter, $\zeta$ and $\omega$, can be tuned to follow the backlash inverse as closely as possible, while utilizing the maximum actuator capacity. The resulting inverse also efficiently rejects any disturbance effects. Furthermore, as shown by the dash dot black line in Fig 2, it only smoothes out the corners required for transition, and thus maintains higher accuracy in compensating for the backlash. The overall implementation of the smooth inverse can be expressed as:

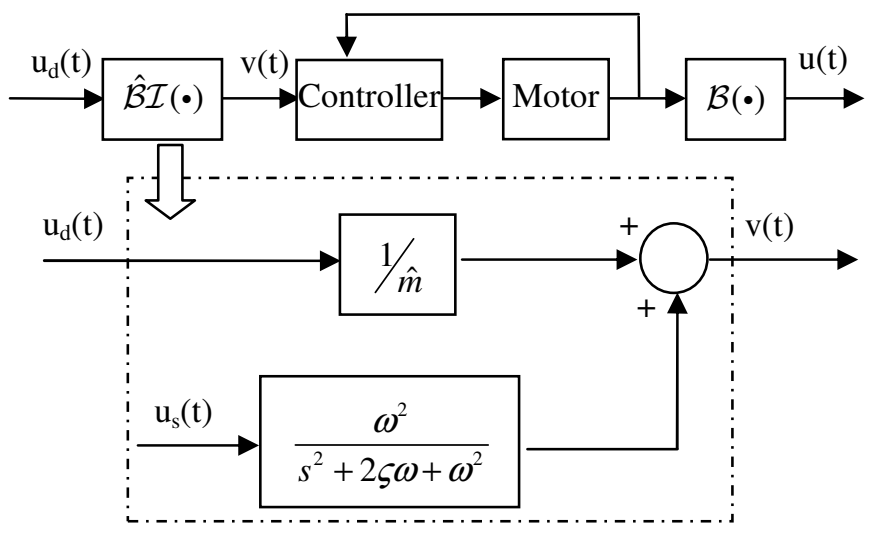

Fig. 3: Implementation of backlash inverse

\section{Feedforward Control}

While a PID controller can lead to limit cycles [25], an appropriately implemented smooth backlash inverse is stable. If both the input and the output to the backlash are available for measurements, backlash parameters can be easily and accurately estimated offline as well as online. And if the precise backlash parameters are known, backlash inverse can be used to compensate for the backlash, as long as the actuator dynamics are fast enough compared to the overall system bandwidth.

It has been shown in [13] that the transmission characteristics in cable actuated systems using pull-pull configuration follow a backlash type of profile, dependent on physical parameters of the system like conduit curvature, pretension, cable length and stiffness. As a simplification, the motion profile can be characterized as a backlash, with time varying slope and offset, thus, effectively representing the physical parameters by the corresponding backlash parameters. For example, for systems using polymeric cables, due to viscoelasticity of the cables, backlash width continuously decreases [19]. Thus, an online parameter estimate which can constantly update the backlash parameters can fit the motion transmission profile. For the simple cases, with a constant backlash, offline parameter estimation can also be utilized. For parameter estimation, following the approach of [20], two indicator functions are defined as:

$\mathcal{X}_{r}(t)=\left\{\begin{array}{cc}1 & \dot{u}(t)>0 \\ 0 & \dot{u}(t) \leq 0\end{array} ; \quad \mathcal{X}_{l}(t)= \begin{cases}1 & \dot{u}(t)<0 \\ 0 & \dot{u}(t) \geq 0\end{cases}\right.$

With these indicator functions as regressor terms, backlash can be expressed by the following equation:

$u(t)=\left[\begin{array}{lll}v(t) & \mathcal{X}_{r}(t) & \mathcal{X}_{l}(t)\end{array}\right] \cdot\left[\begin{array}{lll}m & -m c_{r} & -m c_{l}\end{array}\right]^{T}: \dot{u}(t) \neq 0(7)$

Using (7), any parameter estimation approach, for example least squares method, can be used to estimate backlash parameters. However, backlash parameters are only estimated, when the output is moving, i.e. $\dot{u}(t) \neq 0$. 
Therefore, depending on the direction of motion, only two parameters are estimated at a time.

Thus, for the cable actuated systems, if the measurement of the motion of the remote joint is available, an open loop feedforward controller using smooth backlash inverse can effectively compensate for the nonlinearities due to friction and cable compliance.

\section{EXPERIMENTAL RESULTS}

To study the effectiveness of the smooth backlash inverse, as well as to analyze the system performance using feedforward loop control in surgical instruments, experiments were conducted on two different setups available in lab, and corresponding results are presented in the following sub-sections.

\section{A. Laprotek robot arm}

Experiments were carried out using a robot arm for the Laprotek surgical robot shown in Fig. 4. The arm is designed to have seven degrees of freedom. However, to avoid any coupling effects between various joints, only one of the joints was controlled shown by the dotted circle in Fig 4. For higher precision, the actuating motor was controlled using a dSpace controlled board, instead of the built in microcontroller designed for the robot. The drive motor was Maxon DC brush motor, with a gear head ratio 27.6 and a 500 count encoder, which can measure rotation with an accuracy of $0.18^{\circ}$. Motion of the arm joint was measured using an external encoder with an accuracy of $0.025^{\circ}$. The drive motor was run in position control mode using a PID controller. The actuation cables were nylon coated stainless steel $7 \times 19$ wire rope with $0.58 \mathrm{~mm}$ diameter and approximately $1.25 \mathrm{~m}$ in length. The stainless steel conduits were made from $0.49 \mathrm{~mm}$ diameter wire wrapped into a close packed spring with an inner diameter of $1.29 \mathrm{~mm}$.

Backlash in the system due to the motor gearhead was deemed negligible as compared to the friction effects in the conduit. All the results reported for the actuator motion is the motion of the gearhead shaft, thus gear ratio has been taken into account. A desired trajectory of sinusoidal motion of amplitude $10^{\circ}$, at $0.1 \mathrm{~Hz}$ frequency was applied. Experiments were carried out for three different cases, no backlash inverse, exact backlash inverse and proposed smooth backlash inverse. For the smooth inverse, natural frequency $\omega=50 \mathrm{rad} / \mathrm{sec}$ and damping ratio $\zeta=1$ was used.

Fig. 5(a) shows the backlash present in the system. Fig. 5(b) shows the tracking errors of the remote joint, and the actuating motor for the three cases. Solid black line in Fig. 5(b) shows the error for the case when no backlash inverse is implemented, and instead a constant gain (from input to output) is assumed. Without any compensation, a large error of about $2^{\circ}$ is observed, which translates to a backlash distance approximately twice the tool tip size. Dashed red line in Fig. 5(b) shows the performance of the system when an exact inverse is implemented. The performance improves significantly and the maximum error reduces by half $\left(1^{\circ}\right)$. However, the sudden jumps in the actuator command trajectory lead to large errors in actuator motion.

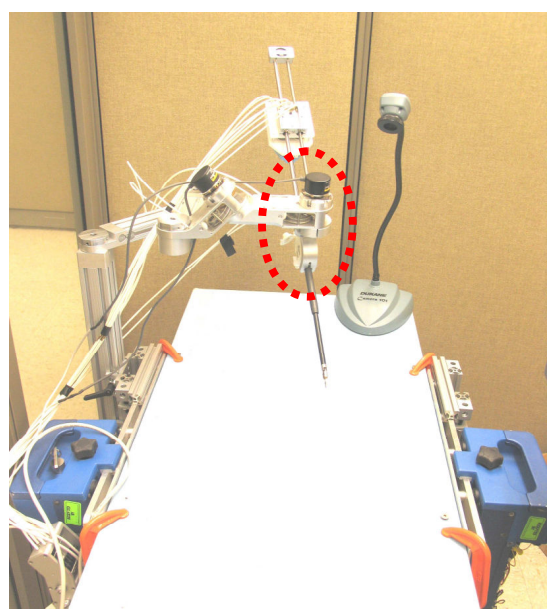

Fig. 4 Patient side robot Arm for Laprotek surgical robot

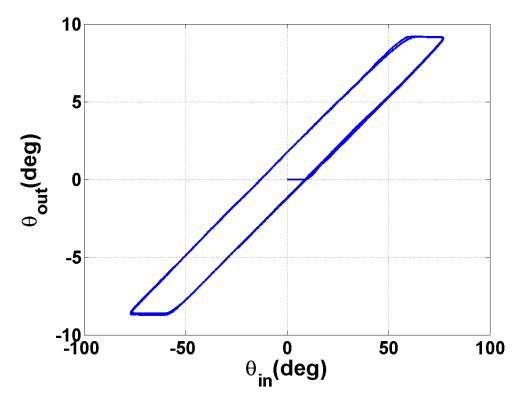

(a) Backlash in the Laprotek robot arm
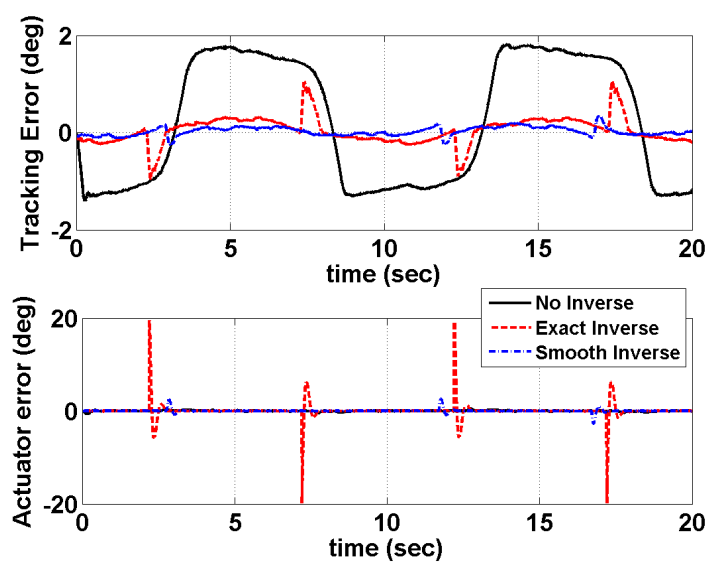

(b) Tracking error in the remote joint motion and the actuator motion

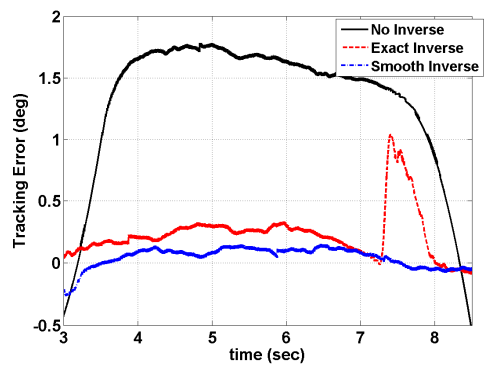

(c) Error during $3 s$ to $8.5 s$

Fig. 5: Experimental results for the robotic arm comparing the system performance without backlash inverse, and with exact and smooth inverse 
Dash-dot blue line in Fig. 5(b) shows the results for the smooth inverse. The error magnitude reduces considerably, as shown in the enlarged plot of tracking error in Fig. 5(c). Smoothing completely removes the large error at the transition points, and although exact inverse would theoretically perform better, in practice properly designed smooth inverse yields better results, and the tracking errors reduces to $0.2^{\circ}$, which translates to approximately $0.1 \mathrm{~cm}$ error at the tool tip.

\section{B. System using Viscoelastic Cables}

Instead of using steel cable-conduits for actuation, polymeric cables passing through PEEK conduit can be used for developing MRI compatible systems [19]. However, instead of repetitive behavior observed for steel cables, using the polymeric cables lead to time varying behavior induced by cable viscoelasticity, thus generating a hysteresis type of transmission profile. As discussed earlier, this can be approximated as a backlash with time-varying parameters, and therefore can be compensated with online parameter estimation. An online recursive least squares approach was used to estimate backlash parameters.

The experimental setup emulates a surgical robot and is shown in Fig. 6. The details of the setup can be found in [13]. The actuating motor was run in position control mode, while the follower motor was run in torque control mode to simulate the environmental load as torsional spring,. For the experiments, braided fishing line (Spiderwire ${ }^{\circledR}$ Stealth $^{T M}$ ) of diameter $0.3 \mathrm{~mm}$ was chosen as actuation cable and was wrapped around $12 \mathrm{~mm}$ diameter motor pulleys. PEEK tubings from Zeus Inc., of inner diameter $0.76 \mathrm{~mm}$, wall thickness $0.2 \mathrm{~mm}$ and length $265 \mathrm{~cm}$ each, were used as conduit. Pulley rotation was measured with a resolution of $0.18^{\circ}$. In the experiment, to emphasize the effect of creep, the desired output trajectory was designed as a slow sinusoid of amplitude $45^{\circ}$, and frequency $0.05 \mathrm{~Hz}$ combined with a trapezoidal pulse input of amplitude $135^{\circ}$ from $80 \mathrm{~s}$ to $190 \mathrm{~s}$, as shown in Fig. 7(a). One and a half loop was introduced in the conduit, and an approximate pretension of $8 \mathrm{~N}$ was applied to the cables.

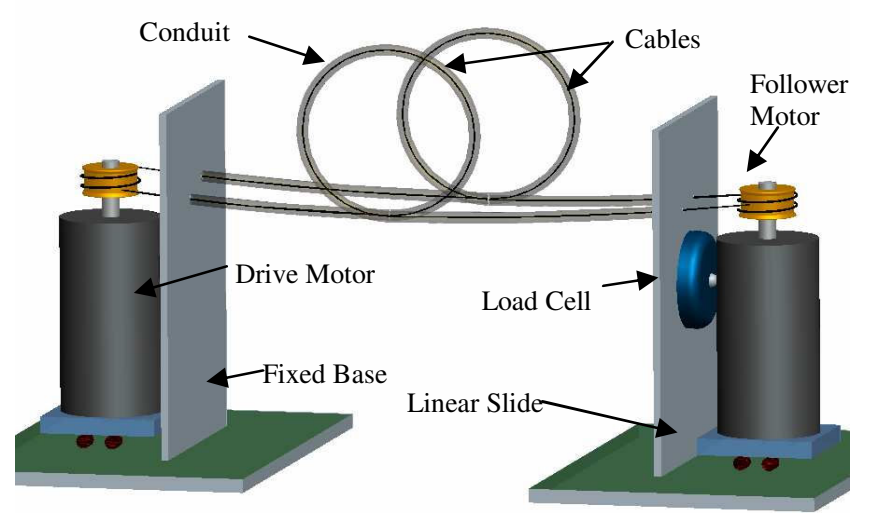

Fig. 6: Experimental setup emulating surgical robot
Figure 7(b) shows the backlash present in the system. The backlash width and slope changed after the implementation of the trapezoidal pulse signal. The solid blue line in Fig. 7(c) shows the tracking error when no backlash inverse was implemented. Without any compensation of the nonlinearity, error magnitudes as high as $25^{\circ}$ can be observed for a desired trajectory amplitude of $45^{\circ}$. Using the smooth inverse, this error has been effectively reduced to less than $3^{\circ}$ after four cycles, as shown by the dashed red line in Fig. 7 (c), and reduces even further after the pulse. However, due to parameter estimation errors, the tracking error does not converge to zero. This is not unexpected as no closed loop control has been implemented. The online backlash parameters estimates are shown in Fig. 7(d).
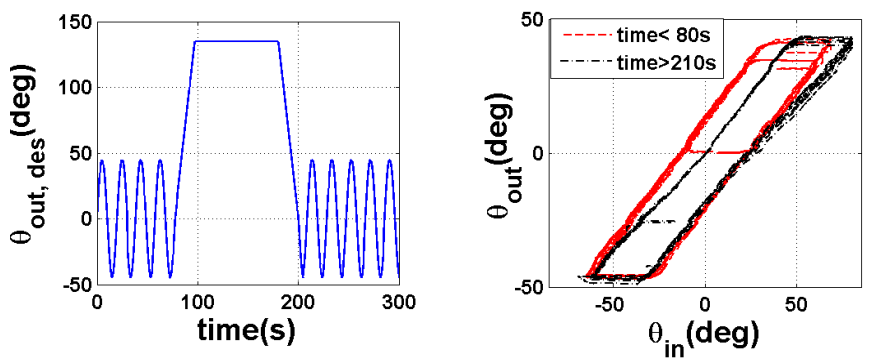

(a) Desired trajectory for the drive motor, (b) Backlash in the system

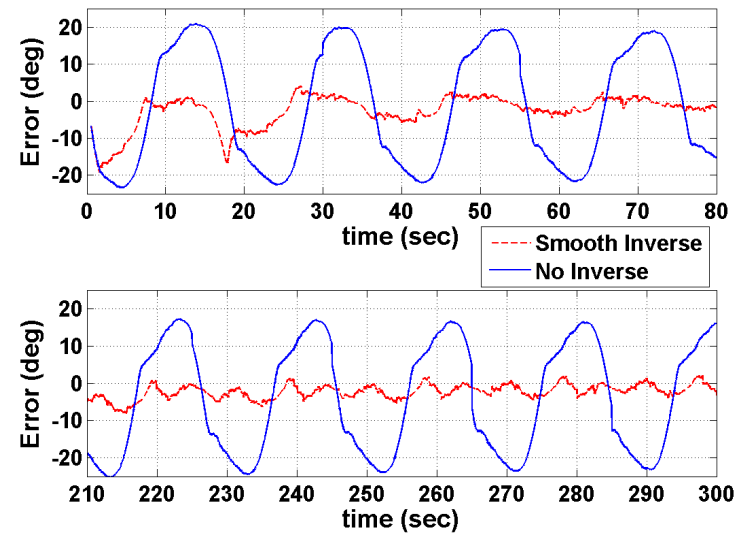

(c) Tracking error of the system with and without inverse implementation, for sinusoidal trajectory before and after the trapezoidal pulse
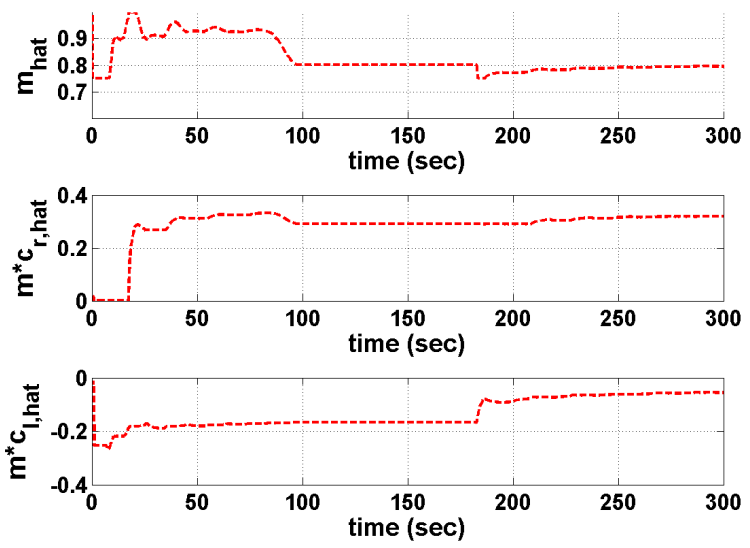

(d) Online backlash parameter estimates

Fig. 7: Experimental results for the system using nylon cables 
As can be observed the parameter estimation process is slower, which leads to larger time duration for the error to settle down. Other parameter estimation approaches like recursive least squares with forgetting factor and covariance resetting need to be considered to make the response faster and to reduce the steady state error. Overall, even without any closed loop control, the error has been reduced considerably to a level where the operator can comfortably adapt in absence of any sophisticated controller.

\section{CONCLUSION}

Cable conduit actuation has various advantages for use in power dense applications like surgical robots, robotic hands and exoskeleton devices. However, it introduces significant nonlinearities in the system and need to be adequately compensated for precise motion control. This paper presents a simple feedforward controller by approximating the nonlinearity as a constant or slowly varying backlash. The use of backlash to model the nonlinearity eliminates the need of correlating the actual physical parameters like pretension and conduit curvature to the motion transmission profile. Backlash parameters can be estimated online at much slower bandwidth compared to the controller implementation, since the parameters change slowly. This provides an advantage of being able to use simple sensors like magnetic trackers, or even image processing methods for position tracking. The paper introduces a novel smooth backlash inverse, designed considering the physical limitations of the actuator, and thus can outperform theoretically better inverse designs. The overall accuracy improvement by the controller using smooth backlash inverse is significant, even for the systems using viscoelastic cables for actuation. In surgical robots, it brings the system at the levels where human perception and deftness of handling the instruments will play a larger role than actuator tracking errors, and the adaptation by the operator will not be as non-intuitive as it would be in the absence of a controller. Eventually for better performance a closed loop control is required, where the controller parameters can be adaptively designed. However, such a design will be largely governed by the accuracy and bandwidth of the sensors available.

\section{REFERENCES}

[1] M. J. H. Lum, D. C. W. Friedman, H. H. I. King, R. Donlin, G. Sankaranarayanan, T. J. Broderick, M. N. Sinanan, J. Rosen, and B. Hannaford, "Teleoperation of a Surgical Robot Via Airborne Wireless Radio and Transatlantic Internet Links" Field and Service Robotics, STAR 42, 2008, pp. 305-314.

[2] G. S. Guthart, and J. K. Salisbury, "The Intuitive telesurgery system: Overview and applications," in Proc. IEEE Int. Conf. Robotics and Automation, San Francisco, CA, April 2000, pp. 618-621.

[3] P. Swain, "The future of colonoscopy". In Colonoschopy principles and practice, 1st ed., J. D. Waye, D. K. Rex, C. B. Williams, Ed. Blackwell Publishing Ltd., pp. 630-638, 2007
[4] G. W. Dachs II and W. J. Peine, "A Novel Surgical Robot Design: Minimizing the Operating Envelope Within the Sterile Field", in Proc. 28th IEEE EMBS Ann. Int. Conf. New York City, Sep. 2006

[5] R.J. Fanzino, "The Laprotek surgical system and the next generation of robotics," Surgical Clinics of North America. vol. 83(6), 2003, pp. 1317-20.

[6] A. Schiele, P. Letier, R. van der Linde, and F. van der Helm., "Bowden Cable Actuator for Force-Feedback Exoskeletons", in Proc. IEEE Int. Conf. on Intelligent Robots and Systems, Beijing, China, Oct 2006, pp 3599-3604

[7] A. Schiele, "Performance Difference of Bowden Cable relocated and non-relocated master actuators in virtual environment applications", in Proc. IEEE Int. Conf. on Intelligent Robots and Systems, Nice, France, Sep 2008, pp 3507-3512

[8] Biagiotti, L., F. Lotti, C. Melchiorri, G. Palli, P. Tiezzi and G. Vassura, "Development of ub hand 3: Early results," in Proc. IEEE Int. Conf. on Robotics and Automation (ICRA 2005), Barcelona, Spain, April 2005, pp. 4488-4493.

[9] F. Lotti and G. Vassura, "A novel approach to mechanical design of articulated finger for robotic hands," in Proc. IEEE/RSJ Int. Conf. on Intelligent Robots and Systems (IROS 2002), Lausanne, Switzerland, October 2002, pp. 1687-1692

[10] N. Ali, J. M. Hollerbach, Y. Xu and I. W. Hunter, "Investigation of the transmission system of a tendon driven robot hand," in Proc. IEEE Int. Conf. on Intelligent Robots and Systems (IROS 1994), Munich, Germany, September 1994, vol. 1, pp. $202-208$

[11] M. Kaneko, W. Paetsch and H. Tolle, "Input-dependent stability of joint torque control of tendon-driven robot hands," IEEE Trans. Industrial Electronics, vol. 39(2), April 1992 pp. 96 - 104.

[12] W.T. Townsend and J.K. Jr. Salisbury, "The effect of coulomb friction and stiction on force control," in Proc. IEEE Int. Conf. on Robotics and Automation, vol.4, Mar 1987, pp 883-889.

[13] V. Agrawal, W.J. Peine, B. Yao, "Modeling of Closed Loop CableConduit Transmission System", in Proc. IEEE Int. Conf. on Robotics and Automation, Los Angeles, CA, May 2008, pp 3407-3412.

[14] P. Letier, A. Schiele, M. Avraam, M. Horodinca and A. Preumont, "Bowden Cable Actuator for Torque-Feedback in Haptic Applications" in Proc. Eurohaptics 2006 Conf., Paris, July 2006.

[15] M. Kaneko, M. Wada, H. Maekawa and K. Tanie, " A new consideration on tendon tension control system of robot hands," in Proc. 1991 IEEE Int. Conf. on Robotics and Automation, Sacramento, 1991, vol.2, pp 1028 - 1033

[16] M. Kaneko, T. Yamashita and K. Tanie, "Basic considerations on transmission characteristics for tendon drive robots," Int. Conf. on Advanced Robotics, vol. 1, 1991, pp. $827-832$ [ $5^{\text {th }}$ Annual. Conf. Robots in Unstructured Environments, 1991]

[17] G. Palli and C. Melchiorri, "Model and control of tendon-sheath Transmission Systems," Proc. IEEE Int. Conf. on Robotics and Automation, 2006

[18] G. Palli and C. Melchiorri, "Optimal Control of Tendon-Sheath Transmission Systems," IFAC symposium of Robot Control, 2006

[19] V. Agrawal, W. J. Peine and B. Yao, "Modeling of Transmission Characteristics for MRI Compatible Viscoelastic Cable-Conduit System", submitted to IEEE ASME Transactions on Mehcatronics.

[20] G. Tao and P. V. Kokotovic, "Adaptive Control of Systems with Sensors and Actuator Nonlinearities", John Wiley and Sons, Inc., 1996

[21] J. Zhou \& C. Wen, "Adaptive Backstepping Ccontrol of Uncertain Systems", Springer-Verlag Berlin Heidelberg, 2008, pp. 83-96.

[22] M. Nordin and P. O. Gutman, "Controlling mechanical systems with backlash - a survey," Automatica, vol. 38, 2002, pp. 1633-1649.

[23] G. Tao and F. L. Lewis, "Adaptive Control of Nonsmooth Dynamic Systems," Springer New York, 2001.

[24] A. Taware and G. Tao, "Control of Sandwich Nonlinear Systems," Springer-Verlag, Berlin, 2003

[25] R. S. Barbosa and J. A. Tenreiromachado, "Describing Function Analysis of Systems with Impacts and Backlash," Nonlinear Dynamics, vol. 29, 2002, pp. 235-250. 